\title{
THE EFFECT OF DIFFERENT SURFACE TREATMENTS ON SHEAR BOND STRENGTH OF CUBIC ZIRCONIA
}

\author{
Engy Azmy Hemdan*, Tarek Salah Morsi* and Fatma Adel ${ }^{*}$
}

\begin{abstract}
Statement of the problem: The conventional methods of adhesive bonding used in silica containing ceramics cannot be used for bonding of zirconia due to absence of silica.

Objective: The study was designed to evaluate the effect of different surface treatments on the shear bond strength of cubic zirconia before and after aging.

Materials and Methods: A total of 56 cubic zirconia plates with dimensions (10mm length and $10 \mathrm{~mm}$ width and $2 \mathrm{~mm}$ thickness) were divided into four groups according to surface treatment (Group 1; No treatment $\mathrm{n}=14$, Group 2; Air abrasion 50 $\mu \mathrm{m}$ AL2O3 particles for 15 seconds $\mathrm{n}=14$, Group 3; CO2 laser at 10W for 10 seconds n=14, Group 4; CO2 laser at $20 \mathrm{~W}$ for 10 seconds $n=14$ ), then each group was subdivided into 2 subgroups which are(Group a: before aging) and (Group b: after aging) $n=7$. All specimens were cemented with MDP resin cement (Panavia SA cement plus) and stored in a distilled water for 24 hours. Then shear bond strength test (SBS) was assessed in $\mathrm{MPa}$ with a universal testing machine before and after thermocycling.
\end{abstract}

Results: The results of this study clearly showed that the samples treated with $\mathrm{CO}_{2}$ laser groups at $20 \mathrm{~W}$ have the highest shear bond strength of resin cement to zirconia ceramic followed by $\mathrm{CO}_{2}$ at $10 \mathrm{~W}$ followed by sandblasting group and the control group showed the least shear bond strength.

Conclusion: The application of $\mathrm{CO}_{2}$ laser surface treatment enhanced the shear bond strength of ultra-translucent zirconia to MDP containing resin cement.

KEYWORDS: cubic zirconia, $\mathrm{MDP}, \mathrm{CO}_{2}$ laser.

\section{INTRODUCTION}

The introduction of computer-aided-design and manufacturing (CAD/CAM) technology provided us with high strength ceramics like zirconia with flexural strength 900-1200 MPA. Zirconium dioxide $\left(\mathrm{ZrO}_{2}\right)$ is by addition of modified yttria
$\left(\mathrm{Y}_{2} \mathrm{O}_{3}\right)$ tetragonal polycrystal (Y-TZP). The addition of Yttria is for stabilizing the crystal structure transformation during firing at high temperature and for improved the physical properties of zirconia. ${ }^{(1)}$

The major complication of zirconia is their lack of translucency and their tenacious need for veneering

\footnotetext{
* Department of Fixed Prosthodontics, Faculty of Dentistry, Ain Shams University
} 
and this increase the tendency of chipping and cracking. So, by the introduction of nano- zirconia that allow us to overcome the polycrystalline birefringence barrier and to manufacture a translucent, high strength monolithic restorations, like ultra-translucent zirconia that showed a significantly higher degree of translucency, which improved the esthetics. The higher translucency is obtained by changing the yttria $\left(\mathrm{Y}_{2} \mathrm{O}_{3}\right)$ content ( $5 \mathrm{~mol} \%$ or more instead of the conventional 3 mol\%), which is used to stabilize the tetragonal zirconia phase, resulting in a higher amount of cubic phase particles more than $50 \%$. $^{(2,3)}$

Conventional cementation techniques with zirconia ceramics are not able to provide sufficient bond strength due to lack of silica. The strong resin bonding depends on the proper selection of the type of surface treatment and type of resin to achieve micromechanical interlocking and adhesive chemical bonding to the ceramic surface. ${ }^{(1,2)}$ The surface of zirconia is not able to be etched by HF like other ceramics due to absence of silica components, so mechanical and chemical methods have been required to achieve stable bond between zirconia and resin cement. It was reported that the use of resin cements or primers containing MDP enhanced the strength of adhesion by providing chemical retention to zirconia. ${ }^{(4,5)}$ Previous studies have been showed that the zirconia bonded with resin cement using phosphate containing primer like MDP produced a higher durable bond strength compared to MDP free resin systems. ${ }^{(6,7)}$

Different surface treatments have been recommended in order to increase surface roughness and obtain better mechanical bond strength. Airborne particle abrasion has been proposed to facilitate the micromechanical retention between the zirconia ceramics and MDP containing resin cements. This method increasing the surface roughness and surface energy, moreover air abrasion may generate hydroxyl groups on zirconia surfaces facilitating the chemical reaction with phosphate monomers. ${ }^{(8)} \mathrm{On}$ the other hand, air abrasion may cause flaws and cracks and compromise the mechanical properties and long term durability of zirconia. ${ }^{(9)}$

Lasers have been used for many purposes in dentistry such as conditioning of tooth structure or restorative surfaces, different lasers such as $\mathrm{CO}$, Er:YAG, and Nd:YAG, have been used for surface treatment of zirconia ceramics, and showed varying degree of sucsses. The CO2 laser is generally employed for intraoral soft tissue surgery because of its great absorption. The wavelength of the CO2 laser (10600nm) is also well absorbed by ceramic materials, making it a suitable instrument for ceramic surface treatment. ${ }^{(10)}$ It was reported in a previous study that $\mathrm{CO} 2$ laser achieved the highest bond strength values compared by Er:YAG and air abrasion by enhancing micromechanical retention between adhesive and zirconia through thermochemical ablation. ${ }^{(1)}$ However, other study showed contradictory result as CO2 laser surface treatment has been showed lower bond strength compared to Nd;YAG. ${ }^{(12)}$ Nevertheless, the best pre-treatment for zirconia is still controversial.

The purpose of this study is to evaluate the effect of different surface treatments which are Sandblasting and fractional $\mathrm{CO} 2$ laser techniques on the shear bond strength between cubic zirconia and MDP containing resin cement before and after aging.

\section{METHODOLOGY}

\section{METHODS}

56 plates of ultra translucent zirconia with square shape dimensions $(10 \mathrm{~mm}$ length and $10 \mathrm{~mm}$ width and $2 \mathrm{~mm}$ thickness) were obtained by cutting cubic zirconia (Bruxir Anterior, Glidewell laboratories) blank by using a water cooled low-speed diamond saw (Isomet 4000, Buehler, Dusseldorf Germany). The specimens were cut $20 \%$ larger than the 
required dimensions to consider its shrinkage and achieve the needed dimensions (10mm length, $2 \mathrm{~mm}$ thickness) after sintering process. The zirconia plates were sintered in a high temperature furnace according to the manufacturer's recommendations.

Acrylic resin bases were made to hold zirconia plates. Molds were made from polyvinylchloride (PVC) water pipes which were cut into $(25 \mathrm{~mm}$ internal diameter $+20 \mathrm{~mm}$ length). Acrylic resin was mixed according to the manufacturer instructions, the powder/ liquid ratio is $3: 1$. PVC molds and then the acrylic resin mix was poured inside the molds, zirconia plates were placed in the acrylic resin and left to set. Then, the acrylic base was pushed from the tube then finished for excess removal.

Sandblasting unit:(Renfert) sandblasting unit was used for treatment of zirconia plates The specimens were air abraded with $50 \mu \mathrm{m} \mathrm{Al} 2 \mathrm{O} 3$ particles for 15 seconds at a distance of $10 \mathrm{~mm}$ with a pressure of 2.5 bar. In order to standardize the distance between the surface of the plate and the nozzle, the nozzle was stabilized on a customized holder at $10 \mathrm{~mm}$ distance, the distance was determined by using a ruler. The holder was used to hold the nozzle to be perpendicular on the plate during air abrasion, while the nozzle was stabilized on the holder the plate was moving up and down facing the nozzle at the standerdized distance. After air abrasion the specimen was rinsed thoroughly under tap water to remove AL203 particles and air dried.

Laser groups: laser unit (DEKA M.E.L.A. s.r.l. Vla Baldanzese 17-50041 calenzano (F1) Italy) was used for treatment zirconia plates with $\mathrm{Co} 2$ laser at two different parameters which are $10 \mathrm{~W} / 14 \mathrm{~mJ}$ and $20 \mathrm{~W} / 10 \mathrm{~mJ}$. The zirconia plate was stabilized manually and the laser was run in a continuous mode and the tip of laser which is $3 \mathrm{~cm}$ long was held manually perpendicular to the zirconia surface. Movements were performed in lateral direction with frequency $200 \mathrm{HZ}$ (pulse per second) and irradiation time $10 \mathrm{sec}$, then all specimens were ultrasonically cleaned in de-ionized water bath for 10 minutes and gently air- dried.

The resin was applied by using plastic tubes (catheters) with internal diameter $2 \mathrm{~mm}$ and sectioning them to $2 \mathrm{~mm}$ height then the tubes were stabilized on the plates by applying bonding agent on the peripheries of the tubes with micro brush and the resin was applied by using auto mix tip then the specimen was light cured with a high intensity LED device for $40 \mathrm{sec}$. . The plastic tubes were cut with a scalpel blade and removed. The bonded specimens were stored in distilled water for 24 hours before the shear bond strength test.

Half of each group ( $\mathrm{n}=7$ ) was subjected to thermocycling(The 100 SD Mechatronic thermocycler) for 5000 cycles between 5 and $55^{\circ} \mathrm{C}$. The dwell time at each temperature was 30 seconds and the transfer time was 2 seconds.

The specimens were tested in a universal testing machine (Instron universal testing machine, Model 3345 , England). The shear force was applied parallel to the interface of the bonding surface at a crosshead speed of $1 \mathrm{~mm} / \mathrm{min}$ by using a mono-beveled chisel shaped metallic rod, until bonding failure of the specimens occurred.

The maximum force needed for de-bonding was recorded and the bond strength was calculated by dividing the maximum force over the area of the bonding surface. The load-deflection curves were recorded using computer software.

$$
\text { S.B.S }=\mathrm{F} / \mathrm{A}
$$

Where $\mathrm{S}=$ shear bond strength $\left(\mathrm{N} / \mathrm{mm}^{2}\right)$ $\mathrm{F}=$ Maximum load until separation $(\mathrm{N}) \mathrm{A}=$ Surface area of bonding $\left(\mathrm{mm}^{2}\right)$.

To detect surface topography for the untreated and treated cubic zirconia either with sandblasting and $\mathrm{CO} 2$ laser each specimen was coated with gold sputter coat and evaluated under SEM(QUANTA 
FEG 250) at 5000X and 10000X magnification power to asses the changes in surface topography.

After shear bond strength test, samples were examined using digital microscope to determine the mode of bond failure. The images were captured at magnification of $15 \mathrm{x}$ then transferred to IBM personal computer which equipped with image-tool software. Failure mode was categorized as adhesive failure at the zirconia/resin interface, cohesive failure within the resin cement or zirconia itself and mixed failure which is mixed between adhesive and cohesive failure.

\section{RESULTS}

Before aging the significantly highest bond strength showed with the plates treated with $\mathrm{CO} 2$ laser at $20 \mathrm{~W}$ followed by $\mathrm{CO} 2$ at $10 \mathrm{~W}$, sandblasting showed lower SBS and control group showed the least SBS. After aging, it is interesting to note that the effect of thermocycling either with air abrasion or $\mathrm{CO} 2$ laser treatment at $10 \mathrm{~W}$ showed no statistically significant change in mean shear bond strength. While for Laser $20 \mathrm{~W}$ group; there was a statistically significant decrease in mean shear bond strength after aging. as showed in table (1).

The failure mode was totally adhesive in the control group, predominantly adhesive in sandblasting group and predominantly mixed in the laser group without any cohesive mode of failure, as showed in table (2).

TABLE (1): The mean, standard deviation (SD) values and results of two-way ANOVA test for comparison between shear bond strength $(\mathrm{MPa})$ before and after aging with each surface treatment

\begin{tabular}{|l|c|c|c|c|c|c|c|c|}
\hline \multirow{2}{*}{ Aging } & \multicolumn{2}{|c|}{ Control } & \multicolumn{2}{c|}{ Sandblasting } & \multicolumn{2}{c|}{ Laser 10 W } & \multicolumn{2}{c|}{ Laser 20 W } \\
\cline { 2 - 10 } & Mean & SD & Mean & SD & Mean & SD & Mean & SD \\
\hline Before aging & 9.3 & 1.4 & 13.3 & 1.3 & 18.6 & 2.1 & 19.5 & 3.2 \\
\hline After aging & 7 & 1.4 & 12.6 & 2 & 16.3 & 3.1 & 16.7 & 2.9 \\
\hline$P$-value & \multicolumn{2}{|c|}{0.105} & \multicolumn{2}{|c|}{0.582} & 0.078 & $0.027 *$ \\
\hline Effect size (Partial eta squared) & \multicolumn{2}{|c|}{0.056} & \multicolumn{2}{|c|}{0.007} & \multicolumn{2}{|c|}{0.066} & \multicolumn{2}{c|}{0.102} \\
\hline
\end{tabular}

*: Significant at $P \leq 0.05$

TABLE (2): The frequencies (n), percentages (\%) and results of Chi-square and Fisher's Exact test for comparison between modes of failure in different groups

\begin{tabular}{|c|c|c|c|c|c|c|c|c|c|c|c|}
\hline \multirow[t]{2}{*}{ Aging } & \multirow{2}{*}{$\begin{array}{l}\text { Mode of } \\
\text { failure }\end{array}$} & \multicolumn{2}{|c|}{$\begin{array}{l}\text { Control } \\
(n=7)\end{array}$} & \multicolumn{2}{|c|}{$\begin{array}{l}\text { Sandblasting } \\
\quad(\mathbf{n}=7)\end{array}$} & \multicolumn{2}{|c|}{$\begin{array}{l}\text { Laser } 10 \mathrm{~W} \\
\quad(\mathbf{n}=7)\end{array}$} & \multicolumn{2}{|c|}{$\begin{array}{l}\text { Laser } 20 \text { W } \\
\qquad(\mathbf{n}=7)\end{array}$} & \multirow[t]{2}{*}{$P$-value } & \multirow{2}{*}{$\begin{array}{l}\text { Effect } \\
\text { size (v) }\end{array}$} \\
\hline & & $\mathbf{n}$ & $\%$ & n & $\%$ & $\mathbf{n}$ & $\%$ & $\mathbf{n}$ & $\%$ & & \\
\hline \multirow{2}{*}{$\begin{array}{l}\text { Before } \\
\text { aging }\end{array}$} & Adhesive & 7 & 100 & 4 & 57.1 & 2 & 28.6 & 2 & 28.6 & \multirow{2}{*}{$0.022 *$} & \multirow{2}{*}{0.586} \\
\hline & Mixed & 0 & 0 & 3 & 42.9 & 5 & 71.4 & 5 & 71.4 & & \\
\hline \multirow{2}{*}{ After aging } & Adhesive & 7 & 100 & 5 & 71.4 & 4 & 57.1 & 3 & 42.9 & \multirow{2}{*}{0.183} & \multirow{2}{*}{0.452} \\
\hline & Mixed & 0 & 0 & 2 & 28.6 & 3 & 42.9 & 4 & 57.1 & & \\
\hline
\end{tabular}

*: Significant at $P \leq 0.05$ 


\section{DISCUSSION}

Monolithic zirconia restorations were introduced to overcome the problem of porcelain fracture in porcelain veneered zirconia-based restorations. Delamination and chipping stated to be the most common modes of failure. ${ }^{(13,14)}$ Ultra-translucent zirconia has a remarkably higher degree of translucency which providing a greatly improved esthetics and a greater light transmission but with reduced mechanical properties compared with Y-TZP zirconia. It has a flexural strength of 700 to $800 \mathrm{MPa}$ and fracture toughness of 2.2 to $4 \mathrm{MPa}$ $\mathrm{M}^{1 / 2}$. Its specific properties make it an alternative material for the esthetic zone. ${ }^{(15,16)}$ Bonding to zirconia ceramic material has become a matter of interest in recent years, as the traditional method of surface treatment of glass ceramics are not effective on $\mathrm{ZrO} 2$ surfaces, because zirconia is inert, lack silica and glass phase ${ }^{(4,17)}$.

In this study surface treatment with $\mathrm{CO} 2$ at $20 \mathrm{~W}$ showed the statistically significant highest shear bond strength followed by CO2 laser at $10 \mathrm{~W}$. Sandblasting showed statistically significantly lower mean value. Control group showed the statistically significant lowest mean shear bond strength

The results of this study clearly showed that the surface penetration with $\mathrm{CO} 2$ laser increased the shear bond strength of resin cement to zirconia ceramic. This results could be attributed to complete absorption of the energy of $\mathrm{CO} 2$ laser beam. After absorption of laser energy by zirconia ceramic, a process of heat induction produces shell- like ruptures on the zirconia surface which can provide a micromechanical bond between the resin cement and the zirconia surface after resin tags penetrate into these cracks and set. These results was justified by SEM before resin bonding that showed wide cracks, localized melting areas. While after shear test SEM showed resin remnants which represent mixed failure and spherical shape prominences over the surface. Cracks and irregularities were observed as the power increased.
This result is in agreement with Farzaneh Ahrari et al ${ }^{(18)}$ who found that The SBS values of the specimens treated with the fractional $\mathrm{CO}_{2}$ laser at either $10 \mathrm{~W} / 14 \mathrm{~mJ}$ or $20 \mathrm{~W} / 10 \mathrm{~mJ}$ were higher than any of the other treatment groups. This authors attributed this to the efficacy of fractional $\mathrm{CO}_{2}$ laser in roughening the bonding surface through the process of thermomechanical ablation, which increases micromechanical retention, thereby enhancing the bond strength at the cement/zirconia interface. Also our study is in agreement with Ural et $\mathbf{a l}^{(\mathbf{1 9 )})}$ who observed highest bond strength values in $\mathrm{CO}_{2}$ laser treated zirconia compared to the control and Er:YAG laser-treated groups. In contrast, Akhavan Zanjani et al ${ }^{(10)}$ reported that air abrasion had a greater efficacy than $\mathrm{CO}_{2}$ and Er,Cr:YSGG lasers in conditioning the zirconia surfaces to enhance their bonding strengths to resin cement.

Airborne-particle abrasion group showed lower bond strength in comparison to the $\mathrm{CO} 2$ laser treated group which is still within the minimum acceptable bond strength value is suggested to be around 13 Mpa, but this value is not applicable for retention of inlays. ${ }^{(20,21)}$ These results are supported by SEM images that had been taken after surface treatment that showed slightly irregular surface with evidence of multiple microcracks and micropores. (10) In addition, He et al ${ }^{(22)}$ mentioned that the air abrasion is un- preferably used with zirconia as it might cause micro fractures that would decease functional strength and lead to premature catastrophic failure due to phase transformation.

Untreated group (control) showed the lowest means of shear bond strength in comparison to that of air abrasion and $\mathrm{CO} 2$ laser treatment. The results are in agreement with Ahn et al ${ }^{(8)}$ who revealed that the un-treated Y- TZP surfaces showed the lowest bond strength, as high incidence of adhesive failure was detected, leaving the material's surface free of any luting material remnants. This is may be due 
to the poor chemical interaction at the interface between components (MDP component in the resin cement and the hydroxyl groups of the zirconia ceramics).

Some of the samples of the control group showed premature failure during thermocycling. Several studies have demonstrated that the bond strength of resin based materials to acid-resistant ceramics, especially for the oxide ceramic, is neither durable nor stable. ${ }^{(23,24)}$ Ozcan et al ${ }^{(25)}$ revealed in their study that the bond strength of the MDP-containing resin cements to untreated zirconia was also not stable after aging conditions, and moreover, the bond strength results decreased dramatically resulting in 0 $\mathrm{MPa}$. This leads to the fact that formation of surface micro-irregularities and surface pre-treatment for zirconia is essential to allow for a durable bond.

Shear bond strength was decreased after thermocycling, as mismatch between the thermal expansion coefficients of the bonded material (ultra translucent zirconia and MDP containing resin cement) may induce stresses at the zirconia- resin interface during thermocycling of the samples and decrease the bond strength. It was significantly decreased with $\mathrm{CO} 2$ laser at $20 \mathrm{~W}$ group due to overheating resulted from increasing the power that leads to formation of deeper cracks wit incomplete penetration of resin cement. ${ }^{(26)}$

Shear bond strength was not significantly decreased after thermocycling with air abrasion and $\mathrm{CO} 2$ laser at $10 \mathrm{~W}$ groups, this might suggest that Panavia SA cement plus could produce a sufficient resin- zirconia bond. Longer aging time might reduce the bond strength further, although Kern et al ${ }^{(27)}$ found no significant difference even after 150 days of water storage and 37500 cycles of thermocycling when MDP-containing resin cement was bonded to zirconia. Ahn et al ${ }^{(8)}$ determined the effect of different primers on the shear bond strength between Y-TZP zirconia and MDP containing resin cement. After water storage and thermocycling, MDP-based products had a high significant bond strength effect than phosphoric acid based metal/ zirconia primer.

Mode of failure was examined in this study to obtain further information about the quality of the bond and the resin cement interface. the failure were totally adhesive in the control group and mostly adhesive in air abrasion group and mixed in $\mathrm{C} 02$ laser groups, without any cohesive failure. ${ }^{(26)}$

\section{CONCLUSION}

The application of $\mathrm{CO} 2$ laser surface treatment resulted in the highest shear bond strength of ultratranslucent zirconia to MDP resin containing resin cement. While sandblasting surface treatment showed lower bond strength values. Micromechanical and chemical bonding are essential for optimum resin bonding with zirconia.

\section{RECOMMENDATIONS}

1. Fractional $\mathrm{CO} 2$ laser improves the bond strength of zirconia to resin cement.

2. Further investigations are needed for zirconia treated with $\mathrm{CO} 2$ laser to evaluate the extensions of the cracks that achieved by the laser surface treatment and the durability of the bond and the mechanical properties of zirconia.

\section{REFERENCES}

1. Vargas, M. A., Bergeron, C. \& Diaz-Arnold, A. Cementing all-ceramic restorations: recommendations for success. J. Am. Dent. Assoc. 2011; 142: 20S-24S.

2. Rosentritt, M., Sikora, M., Behr, M. \& Handel, G. In vitro fracture resistance and marginal adaptation of metallic and tooth-coloured post systems. J. Oral Rehabil. 2004; 31: 675-681.

3. Team, G.Laboratoy. Glidewell laboratories announce nanozirconia technology breakthrough. Bruxzir bl. 2011: 2-14.

4. Thompson, J. Y., Stoner, B. R., Piascik, J. R. \& Smith, R. Adhesion/cementation to zirconia and other non-silicate ceramics: where are we now? Dent. Mater. 2011; 27: 71-82. 
5. Janda, R., Roulet, J.-F., Wulf, M. \& Tiller, H.-J. A new adhesive technology for all-ceramics. Dent. Mater. 2003; 19: 567-573.

6. Blatz MB, Sadan A, Kern M. Resin-ceramic bonding: a review of the literature. Journal of Prosthetic Dentistry. 2003; 89(3):268-74.

7. Foxton, R. M., Cavalcanti, A. N., Nakajima, M., Pilecki, P., Sherriff, M., Melo, L., \& Watson, T. F. Durability of resin cement bond to aluminium oxide and zirconia ceramics after air abrasion and laser treatment. Journal of Prosthodontics: Implant, Esthetic and Reconstructive Dentistry. 2011; 20(2), 84-92.

8. Ahn JS, Yi YA, Lee Y, Seo DG. Shear Bond Strength of MDP- Containing Self-Adhesive Resin Cement and YTZP Ceramics: Effect of Phosphate Monomer-Containing Primers. Biomedicine Research International. 2015; 2015:389234.

9. De Mondonca BC, Negrerios WM, Giannini M. Effect of aluminum oxide sandblasting, plasma application and their combination on the bond strength of resin cement to zirconia ceramics. Brazilian Dental Science.2019; 22(4):275280.

10. Akhavan Zanjani V, Ahmadi H, Nateghifard A, Ghasemi A, Torabzadeh H, Abdoh Tabrizi M, Alikhani F, Razi R, Nateghifard A. Effect of different laser surface treatment on microshear bond strength between zirconia ceramic and resin cement. Journal of investigative and clinical dentistry. 2015; 6(4):294-300.

11. Ural C, Kulunk T, Kulunk S, Kurt M. The effect of laser treatment on bonding between zirconia ceramic surface and resin cement. ActaOdontol Scand. 2010; 68(6):354359.

12. Akin H, Ozkurt Z, Kirmali O, Kazazoglu E, Ozdemir AK. (Shear bond strength of resin cement to zirconia ceramic after aluminum oxide sandblasting and various laser treatments. Photomed Laser Surg. 2011; 29(12):797-802.

13. Chen, Y.-W., Moussi, J., Drury, J. L. \& Wataha, J. C. Zirconia in biomedical applications. Expert Rev. Med. Devices 2016; 13: 945-963.

14. Sailer, I., Gottner, J., Känel, S. \& Franz Hämmerle, C. H. Randomized controlled clinical trial of zirconia-ceramic and metal-ceramic posterior fixed dental prostheses: a 3-year follow-up. Int. J. Prosthodont. 2009; 22: 553-60.
15. Denry, I. \& Kelly, J. R. Emerging ceramic-based materials for dentistry. J. Dent. Res. 2014; 93: 1235-1242.

16. Pardo, N. P., Araya, P. L. \& Pardo, M. P. Effect of different surface treatments on the bonds strength of a resin cement in zirconia frameworks. J. Int. Dent. Med. Res. 2016; 9:1-5.

17. Yenisey M, Dede DÖ, Rona N. Effect of surface treatments on the bond strength between resin cement and differently sintered zirconium-oxide ceramics. Journal of Prosthodontic Research. 2016; 60(1):36-46.

18. Ahrari F, Boruziniat A, Alirezaei M. Surface treatment with a fractional $\mathrm{CO} 2$ laser enhances shear bond strength of resin cement to zirconia. Laser therapy. 2016;25(1):19-26.

19. Ural C, Kulunk T, Kulunk S, Kurt M. The effect of laser treatment on bonding between zirconia ceramic surface and resin cement. ActaOdontol Scand. 2010; 68(6):354-359.

20. Maged M. Mohamed Zohdy and Ahmed Khaled Abo El Fadl. The effect of aging and microshear bond strength of resin cement to monolithic zirconia. 2017; 63(3),0070-9484

21. Dérand P, Dérand T. Bond strength of luting cements to zirconium oxide ceramics. Int J Prosthodont 2000; 13: $131-135$

22. He M, Zhang Z, Zheng D, Ding N, Liu Y. Effect of sandblasting on surface roughness of zirconia-based ceramics and shear bond strength of veneering porcelain. Dental Materials Journal. 2014; 33(6):778-85.

23. Aboushelib MN, FeilzerA, Kleverlaan CJ. Bonding to zirconia using a new surface treatment. Journal of Prosthodontics. 2010; 19(7):340-346.

24. Kern, M. \& Wegner, S. M. Bonding to zirconia ceramic: adhesion methods and their durability. Dent. Mater. 1998; 14: 64-71.

25. Ozcan M, Kerkdijk S, Valandro LF. Comparison of resin cement adhesion to Y-TZP ceramic following manufacturers' instructions of the cements only. Clinical Oral Investing. 2008; 12(3):279-82.

26. Kasraei S, Atefat M, Beheshti M, Safavi N, Mojtahedi M, Rezaei- Soufi L. Effect of Surface Treatment with Carbon Dioxide (CO2) Laser on Bond Strength between Cement Resin and Zirconia. Journal of Lasers Medical Science. 2014; 5 (3):115-20.

27. Kern M, Thompson VP. Sandblasting and silica coating on dental alloys: volume loss, morphology and changes in the surface composition. Dental Materials .2003; 9:155-61 